\title{
Morphological characterization of the human corneal epithelium by in vivo confocal laser scanning microscopy
}

\author{
Katharina A. Sterenczak ${ }^{1 \#} \wedge$, Karsten Winter ${ }^{2 \#}$, Karsten Sperlich ${ }^{1,3}$, Thomas Stahnke ${ }^{1,3}$, Stephan Linke ${ }^{4,5}$, \\ Sanaz Farrokhi ${ }^{4}$, Maren Klemm ${ }^{4}$, Stephan Allgeier ${ }^{6}$, Bernd Köhler ${ }^{6}$, Klaus-Martin Reichert ${ }^{6}$, \\ Rudolf F. Guthoff ${ }^{1,3}$, Sebastian Bohn ${ }^{1,3}$, Oliver Stachs ${ }^{1,3}$ \\ ${ }^{1}$ Department of Ophthalmology, Rostock University Medical Center, Rostock, Germany; ${ }^{2}$ Institute of Anatomy, Medical Faculty, University \\ of Leipzig, Leipzig, Germany; ${ }^{3}$ Department Life, Light \& Matter, University of Rostock, Rostock, Germany; ${ }^{4}$ Department of Ophthalmology, \\ University Medical Center Hamburg-Eppendorf, Hamburg, Germany; ${ }^{5}$ Zentrumsehstärke, Hamburg, Germany; ${ }^{6}$ Institute for Automation and \\ Applied Informatics, Karlsruhe Institute of Technology (KIT), Karlsruhe, Germany
}

"These authors contributed equally to this work.

Correspondence to: Dr. Katharina Sterenczak. Department of Ophthalmology, Rostock University Medical Center, Doberaner Straße 140, D-18057 Rostock, Germany. Email: katharina.sterenczak@med.uni-rostock.de.

Background: Regarding the growing interest and importance of understanding the cellular changes of the cornea in diseases, a quantitative cellular characterization of the epithelium is becoming increasingly important. Towards this, the latest research offers considerable improvements in imaging of the cornea by confocal laser scanning microscopy (CLSM). This study presents a pipeline to generate normative morphological data of epithelial cell layers of healthy human corneas.

Methods: 3D in vivo CLSM was performed on the eyes of volunteers $(\mathrm{n}=25)$ with a Heidelberg Retina Tomograph II equipped with an in-house modified version of the Rostock Cornea Module implementing two dedicated piezo actuators and a concave contact cap. Image data were acquired with nearly isotropic voxel resolution. After image registration, stacks of en-face sections were used to generate full-thickness volume data sets of the epithelium. Beyond that, an image analysis algorithm quantified en-face sections of epithelial cells regarding the depth-dependent mean of cell density, area, diameter, aggregation (Clark and Evans index of aggregation), neighbor count and polygonality.

Results: Imaging and cell segmentation were successfully performed in all subjects. Thereby intermediated cells were efficiently recognized by the segmentation algorithm while efficiency for superficial and basal cells was reduced. Morphological parameters showed an increased mean cell density, decreased mean cell area and mean diameter from anterior to posterior $\left(5,197.02\right.$ to $8,190.39$ cells $/ \mathrm{mm}^{2} ; 160.51$ to $90.29 \mu \mathrm{m}^{2} ; 15.9$ to $12.3 \mu \mathrm{m}$ respectively). Aggregation gradually increased from anterior to posterior ranging from 1.45 to 1.53 . Average neighbor count increased from 5.50 to a maximum of 5.66 followed by a gradual decrease to 5.45 within the normalized depth from anterior to posterior. Polygonality gradually decreased ranging from 4.93 to 4.64 sides of cells. The neighbor count and polygonality parameters exhibited profound depth-dependent changes.

Conclusions: This in vivo study demonstrates the successful implementation of a CLSM-based imaging pipeline for cellular characterization of the human corneal epithelium. The dedicated hardware in combination with an adapted image registration method to correct the remaining motion-induced image distortions followed by a dedicated algorithm to calculate characteristic quantities of different epithelial cell layers enabled the generation of normative data. Further significant effort is necessary to improve the algorithm for superficial and basal cell segmentation.

$\wedge$ ORCID: 0000-0002-8122-4203. 


\begin{abstract}
Keywords: Human corneal epithelium; in vivo confocal laser scanning microscopy (CLSM); automatic cell segmentation; quantitative normative data
\end{abstract}

Submitted Sep 10, 2020. Accepted for publication Nov 17, 2020.

doi: 10.21037 /qims-20-1052

View this article at: http://dx.doi.org/10.21037/qims-20-1052

\section{Introduction}

Modern ophthalmology evolved during the last decades from descriptive slit lamp-based findings to in vivo monitoring on the cellular level. During the past decades imaging technologies such as optical coherence tomography (OCT) became essential for the noninvasive in vivo diagnostics of the living eye-especially for the retinal diagnostics. Addressing the anterior segment (AS) of the eye, the ASOCT technology enables quantitative as well as qualitative imaging including the cornea, limbus, anterior chamber and angle without the use of topical anesthesia (1). Based on advanced laser and spectrometer technologies during the past years, the axial resolution of OCT has been dramatically increased and thickness measurements of single corneal layers are available by ultrahigh-resolution OCT $(2,3)$ as well as visualization of cellular and sub-cellular structures by spectral domain OCT (4). While OCT is very promising for corneal cross-sectional imaging, confocal laser scanning microscopy (CLSM) offers en-face imaging with high optical resolution and depth discrimination at the cellular level (1). Due to its axial resolution, selective intracorneal as well as intraepithelial sectioning can be achieved, and the different corneal epithelial cell-types can be distinguished (5). CLSM is suited for monitoring of the living cornea in its physiological state (6) permitting longitudinal examinations of the same cornea in normal and pathological conditions covering the entire ocular surface including the cornea, bulbar and palpebral conjunctiva and lids $(1,7,8)$.

In general there is a number of CLSM based applications for the corneal diagnostic covering a broad range of applications including corneal nerve degeneration and regeneration, chirurgical interventions, contact lenses, diabetes mellitus (DM), keratoconus, keratomycosis, and ocular surface diseases (OSD) $(1,8)$. The evaluation of the subbasal nerve plexus (SNP) of the cornea might add value to the diagnosis of a variety of diseases including limbal stem cell deficiency (LSCD), DM and infection (1). Until today, many studies have aimed in establishing of morphometric features of the SNP as a sensitive marker for ocular and systemic disorders $(9,10)$.
Regarding OSDs, the cellular characterization of the corneal epithelial cell morphology is becoming increasingly important and recent studies have focused on understanding the microstructure of the epithelial cell layers in the cornea (11-13). Besides OSDs, in LSCD for example, a positive correlation between the reduction of basal cell density and the severity of LSCD was reported and basal cell density could be used as a parameter to measure limbal stem cell function at the early stages of the disease process $(14,15)$. In $\mathrm{DM}$, alterations in corneal innervation and basal cell density were reported and it could be demonstrated that reduced basal cell density correlated with changes in innervation (16).

Given the importance of understanding cellular changes in ophthalmological disorders such as OSDs or cellular changes that occur as secondary effects of diseases or treatment regimens, a CLSM-based 3D imaging and automated quantification pipeline could represent a powerful non-invasive tool providing valuable diagnostic information. CLSM has the ability to resolve structural and functional interrelationships, both temporally and spatially in the cornea. Cellular details of biological processes such as inflammation, wound healing, toxicity, and disease could be evaluated over time and help in the design of therapeutic strategies and the assessment of treatment effectivity.

The present study addresses the growing interest in quantitative data of the corneal epithelium and follows up on our initial study (13) that used 3D scanning techniques with fewer capabilities and required OCT data for epithelial thickness determination. The aim was to demonstrate a CLSM-based 3D in vivo image processing pipeline and an automated software for the morphological characterization and quantification of the different epithelial cell layers of the human corneal epithelium.

\section{Methods}

\section{Confocal microscopy and volume data acquisition}

To perform 3D in vivo CLSM, the Heidelberg Retina Tomograph II (HRTII, Heidelberg Engineering GmbH, 

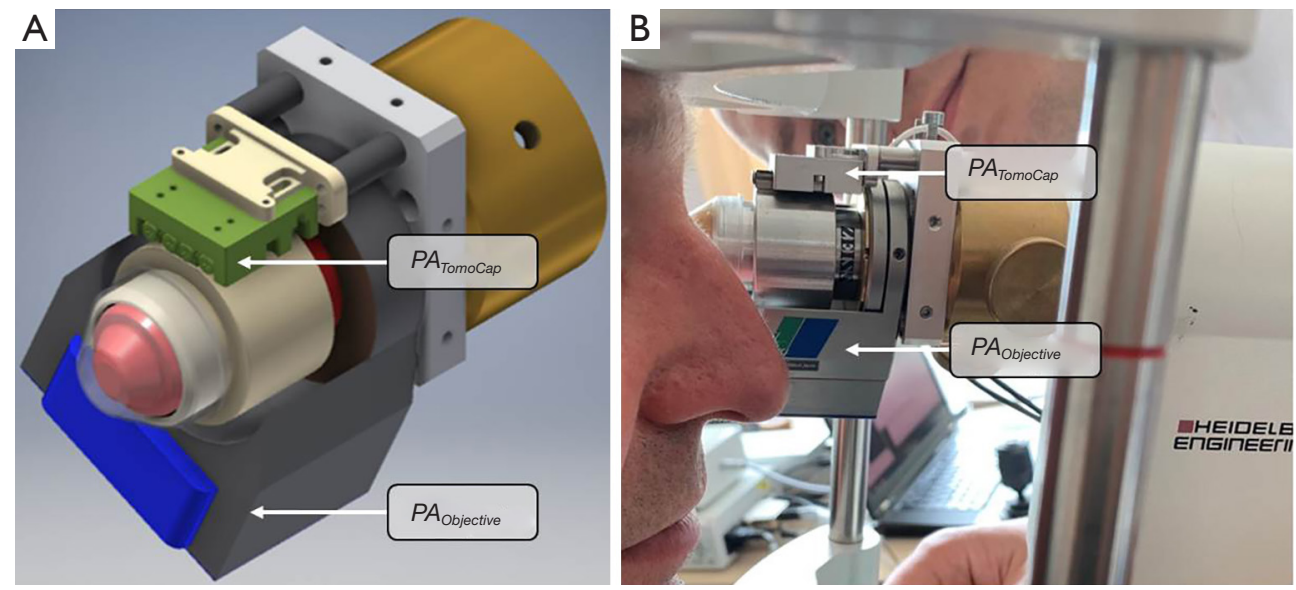

Figure $1 \mathrm{CAD}$ model (A) and patient investigation (B) using the HRTII with an in-house-developed version of the RCM equipped with two piezo actuators: $P A_{\text {Objective }}$ with a closed-loop travel range of $500 \mu \mathrm{m}$ and $P A_{\text {TomoCap }}$ with a rough travel range up to $12 \mathrm{~mm}$ to control the position of a TomoCap. Please note, $P A_{\text {Objective }}$ moves the objective lens and $P A_{\text {TomoCap }}$ the TomoCap.

Heidelberg, Germany) in combination with a modified version of the Rostock Cornea Module (RCM) - RCM 2.0-was used (RCM, Heidelberg Engineering GmbH, Heidelberg, Germany), see Figure 1. The in-house developed modified version of the RCM was previously presented as RCM 2.0 (12). There, the RCM was equipped with a piezo actuator with a closed-loop travel range of $500 \mu \mathrm{m}$ (MIPOS 600 SG OEM, piezosystem jena GmbH, Jena, Germany). This actuator $P A_{\text {Objective }}$ moves a water immersion objective lens (Zeiss, Jena, Germany; 63 x/0.95 w, 670 nm, $\infty / 0)$ and enables a precise focus control. Additionally, the present study introduces a second piezo actuator $P A_{\text {Tomo Cap }}$ (SLC-1720-020-W-L; SmarAct GmbH, Oldenburg, Germany) to control the position of a contact element (TomoCap; Heidelberg Engineering, Heidelberg, Germany) during measurement. This allows the TomoCap to be moved in a controlled manner while in contact with the cornea in order to set the optimal starting focus position for achieving the largest possible height of the cornea stack and to minimize patient's examination time. As suggested in (12), a modified TomoCap is used to reduce lateral eye movements and applanation artifacts. The modified TomoCap has a concave surface with a diameter of $10 \mathrm{~mm}$ and a curvature radius of $7.8 \mathrm{~mm}$, corresponding to the curvature of an average human cornea, thus creating a form closure with the cornea surface during in vivo CLSM (12).

The study included 25 volunteers (average age 44 years, ranging from 25 to 62 years) with normal corneas for investigation by in vivo CLSM. Exclusion criteria were: contact lens wear, keratoconus, and any corneal surgeries in the past. Refraction error was not considered. In accordance with the Declaration of Helsinki, this study was conducted and explained in detail to all volunteers. Informed consent was obtained prior to conducting any investigative procedures.

A carbomer gel (Vidisic; Bausch \& Lomb/Dr. Mann Pharma, Berlin, Germany) was used as the immersion medium. It was inserted between the objective lens and the TomoCap as well as between the TomoCap and the cornea. Before the immersion gel was applied to the cornea, a drop of topical anesthetic (Proparakain-POS; Ursapharm, Saarbrücken, Germany) was instilled. The immersion gel minimizes back reflections from interfaces, enables the high numerical aperture of the objective lens and thus improves the image quality.

The scans were performed in the central cornea and in both eyes of the respective test person. To acquire fullthickness volume data sets of the epithelium, we utilized the $P A_{\text {Tomocap }}$ to position the TomoCap so that the initial focus was slightly outside the cornea. Then, with the $P A_{\text {Objective }}$, the image focus was moved at a constant speed of $30 \mu \mathrm{m} / \mathrm{s}$ through the entire epithelium. During this through-focusing, the HRT acquired the images with 30 frames per second. The field of view of the images was $350 \mu \mathrm{m} \times 350 \mu \mathrm{m}$ at $384 \times 384$ pixels. This resulted in a voxel resolution of $0.91 \mu \mathrm{m} \times 0.91 \mu \mathrm{m} \times 1 \mu \mathrm{m}$ per voxel.

\section{Image registration}

It is well-known that during the fixation of a stationary 
Image processing steps

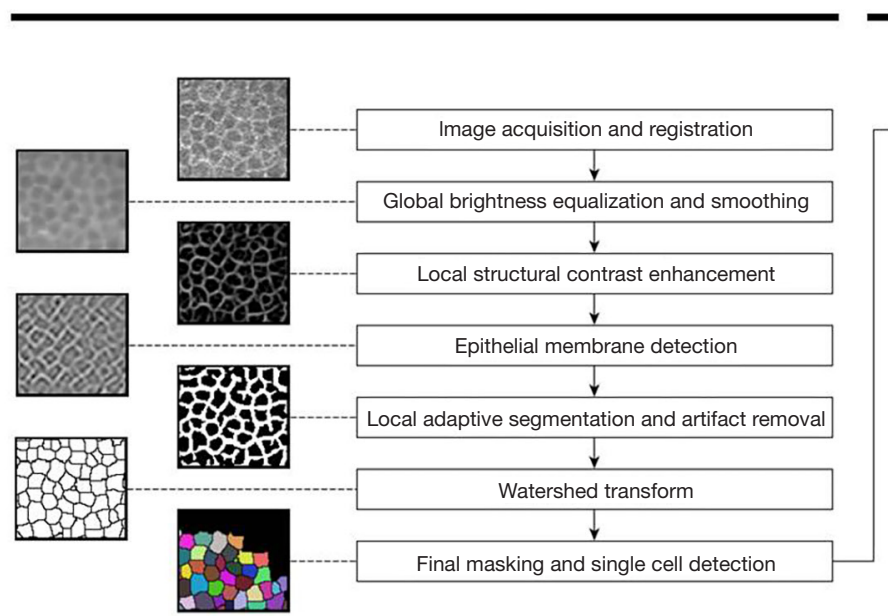

Quantification

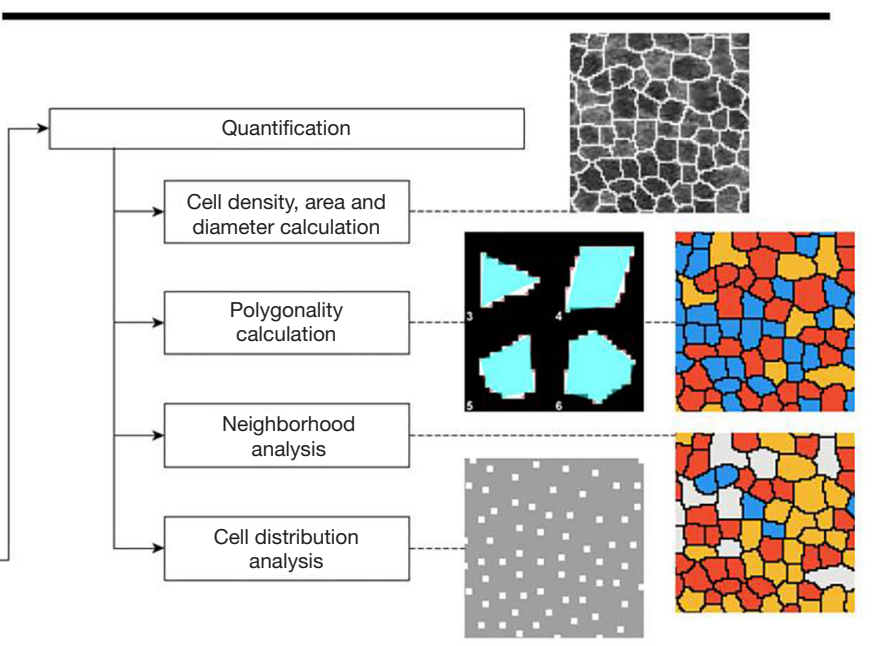

Figure 2 Schematic overview of all image processing (left side) and quantification (right side) steps: Image processing includes all methods needed for the detection of single cells from the original gray-scale images. Single cells were subsequently counted, quantified based on morphological parameters, and analyzed regarding their spatial relations.

target, the human eyes are continuously affected by small involuntary movements, commonly categorized as microsaccades, ocular drifts and ocular microtremors $(17,18)$, ordered by decreasing amplitude. Because of the point-wise scanning of CLSM, these movements cause characteristic image distortion $(13,19)$. The high-velocity microsaccades in particular lead to significant deformation of the cell shapes in the affected image data. Even though the curved contact cap effectively attenuates the eye movements, clearly visible motion artifacts are still present. In order to avoid adverse effects on cell characterization, the same motion-compensating image registration algorithm is used as in the previous study (13). The motion-corrected and aligned image frames are finally stacked to create a nearly isotropic volume representation.

\section{Reconstruction of epithelium, automatic cell segmentation and quantification}

Further image processing and analysis were performed using Mathematica (Version 12.0, Wolfram Research Inc., Champaign, IL, USA). Figure 2 shows a schematic overview of the applied methods. Global brightness equalization was performed on all stack images to compensate varying image illumination ("vignette effect"). Equalized image stacks were smoothed with a $3 \times 3 \times 3$ pixel wide $3 \mathrm{D}$ Gaussian filter $(\sigma=1.5)$ to enhance transitions of cell membranes between adjacent volume slices. For each image slice, image noise was removed and local structural coherence of membranes (and other present structures) was amplified by using a ridgedetecting image filter ("RidgeFilter", $\sigma=1$ ). A bandpass filter for epithelial membrane detection was empirically designed ("BandpassFilter", cutoff frequencies of 0.28 and 1 , filter kernel with a width of 62 pixels) and applied to all preprocessed images. This filter attenuates low (e.g., illumination gradients) and high (e.g., image noise) image frequencies while leaving the mid-range image frequencies (i.e., membranes and structures similar in diameter) unchanged. For each image stack, per pixel bandpass filter responses were summarized for individual images and normalized to respective image sizes. Resulting curves of depth-depending detection values were smoothed with a 3 pixel wide 1D mean filter and an empirically determined threshold value of 0.035 (representing images with still recognizable membranes) was applied to each curve to identify images from the epithelium of the respective image stack. Structure segmentation of all identified epithelial images was performed by local adaptive binarization ( $5 \times 5$ pixel wide window) and small artifacts were removed. Resulting binary images were inverted and a watershed transformation was performed for cell detection. Due to the vignette effect inherent to CLSM images of the cornea, contrast and visibility of the imaged cells decrease significantly towards the image borders. This property results 
in a loss of cell detection quality with increasing distance from image centers. Therefore, a central circular area with a diameter of 250 pixels (corresponding to almost $0.041 \mathrm{~mm}^{2}$ ) was defined around image centers and all cells outside this area were excluded from further processing and analysis.

Cross-sections of all detected epithelial cells were quantified and measurements were averaged for each image slice. Quantities included cell density (cells $\left./ \mathrm{mm}^{2}\right)$, cell area $\left(\mu \mathrm{m}^{2}\right)$ and cell diameter $(\mu \mathrm{m})$. Polygonality (sides) of cells was calculated by approximating polygons to cell shapes using a modified Ramer-Douglas-Peucker algorithm $(20,21)$. The neighborhood count (n) was calculated based on the number of adjacent neighbors of each cell. Finally, cell patterns were analyzed based on the Clark-Evans aggregation index (CEIndex) (22).

For each parameter analyzed, data were separated by age group and functions were fitted within the defined intervals. Detailed ratios of neighborhood count and polygonality of cells across the defined interval were visualized in the form of stacked bar charts.

\section{Results}

\section{Volume data acquisition, image registration}

CLSM imaging with RCM 2.0 using two dedicated piezo stages enabled successful full-thickness volume data collection of the epithelium. Furthermore, the attenuation of the eye movements by the concave TomoCap led to registered image stacks with an increased lateral coverage. The median of the area projected into the $x-y$-plane, in which all registered images of a stack contain information was 45,723 $\mu^{2}$ (min: 27,253 $\mu^{2}$, max: 47,794 $\mathrm{m}^{2}$ ). Additionally, the integrated $P A_{\text {TomoCap }}$ accelerated the entire acquisition procedure by simplifying the adjustment of this cap prior to the start of the acquisition process.

All scans were manually checked for the presence of superficial cells (SCs) and the SNP, for contrast and the signal-to-noise ratio. In total 43 scans of 25 volunteers were used for the following segmentation and quantification procedures. The presence of SCs and the SNP allowed a definition of a normalized depth scale ranging from 0 to 1 (first $\mathrm{SC}=0, \mathrm{SNP}=1$ ) from anterior to posterior. Thereby the comparison of corresponding epithelial cell layers between the data sets independent of the actual epithelial thickness was possible. For the evaluation of a possible age dependency, the 25 volunteers were divided into three age groups: Group 1: $20-35$ years $(\mathrm{n}=10)$; group 2: $36-55$ years $(\mathrm{n}=9)$ and group 3: 56-70 $(\mathrm{n}=6)$.

\section{Reconstruction of the corneal epithelium}

The $3 \mathrm{D}$ reconstruction and segmentation of the corneal epithelial cell layers was performed for all scans (Figure 3). Visual inspection of the image registration results revealed good alignment performance with no visible errors in the regions from the SNP through the basal and intermediate epithelium. Several visible distortion artifacts, mainly caused by overexposed regions, were found in a few superficial images. Individual characteristic cell layers including superficial, upper wing, lower wing-, and basal cells could be reconstructed in 3D (Figure 3B). The developed automatic segmentation algorithm did not work efficiently for superficial and deeper regions of basal cells. Therefore, manual segmentation for one scan was performed to visualize the extreme changes in morphology from anterior (SCs) to posterior (basal cells). Thereby, the mean cell areas ranged from $1,121.8$ to $57.2 \mu^{2}$ and mean cell diameters ranged from 39.5 to $8.9 \mu \mathrm{m}$ (Figure 3C).

\section{Automated segmentation and quantification}

Figure 4 shows an overview of the data collected for the study and used for quantification. Because of individual differences of the epithelial thickness, the interslice distance varied between the datasets when expressed in normalized depth coordinates. Depth dependent cell characteristics could be exemplified for all analyzed scans (Figure 5). The segmentation algorithm worked reliably within the upper and lower wing cell area covering $70-95 \%$ of the available image sections, while the percentage of available image data went down within superficial and basal cell area (Figure 5A).

Segmented cells were color-coded depending on the number of surrounding neighbors (Figure $5 D$ ) and the polygonality (Figure $5 E$ ). Depending on the depth from anterior to posterior, changes of the color-patterns could be observed (Figure 5D,E). The corresponding values are summarized in Figure $5 F$. Depth depending changes were present in the number of surrounding neighbors and the polygonality showing different morphological characteristics.

\section{Repeatability of measurements}

In advance of the study, we have performed repeated scans of volunteers and analyzed the data respectively. An 


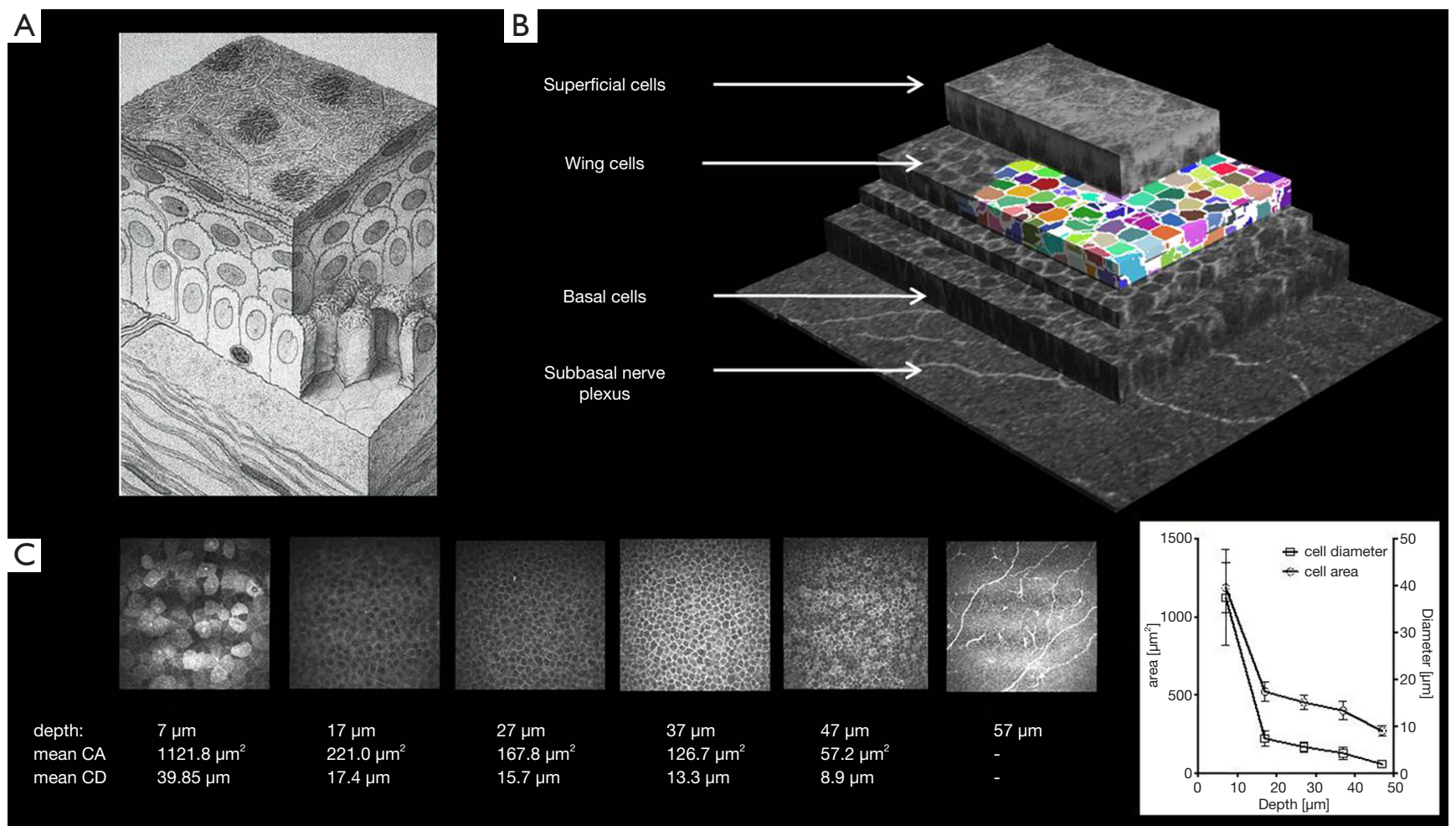

Figure 3 Reconstruction and segmentation of the corneal epithelial cell layers. Corneal epithelium sketch (A) (23) and corneal image stack with cut-outs according to figure (A) recorded with the concave TomoCap in isometric view using KIT-alignment (B) (19). Each of the cells were colored individually after segmentation and 3D reconstruction. (C) Selected images of a stack are shown, exemplifying the typical depth depending cell morphology ranging from the outer superficial cells (depth $7 \mu \mathrm{m}$ ) to basal cells (depth $47 \mu \mathrm{m}$ ). Using manual segmentation, mean cell areas (CA) ranged from $1,121.8$ to $57.2 \mu^{2}$ and mean cell diameters (CD) ranged from 39.85 to $8.9 \mu \mathrm{m}$. Furthermore a graph of the determined values for area and diameter by manual segmentation is shown.

averaged coefficient of variation was determined. In order to exemplify the error range, four independent depth scans of one volunteer were performed and used for automated segmentation, quantification and analysis of variation. The mean coefficient of variation was found for each parameter using the data of successful segmented areas: cell density 0.040; cell area 0.045; cell diameter 0.022; CE-Index 0.005; neighbor count 0.005 ; polygonality count 0.004 .

\section{Generation of normative data}

Figure 6 shows the analyzed quantities for the respective parameters (column A: total mean values; column B: agedependent mean values). In Table 1 the analyzed parameters and in Table 2 total mean values are listed.

The neighborhood and polygonality parameters were additionally evaluated according to their percentage of distribution from anterior to posterior for all analyzed scans (Figure 7). Depth-dependent changes in the number of neighbors and the polygonality of cells were detected confirming the morphological characteristics within the different layers of the corneal epithelium.

\section{Conclusions}

The morphological characterization and quantification as well as the understanding of the proliferative and differentiation behavior of the different corneal epithelial cell layers is of clinical relevance as changes affecting corneal cells and nerves are not only characteristic for unified corneal pathologies but also reflect non-specific pathological processes present in many diseases.

Anatomically, the healthy human corneal epithelium consists of five to six layers of nucleated cells which 


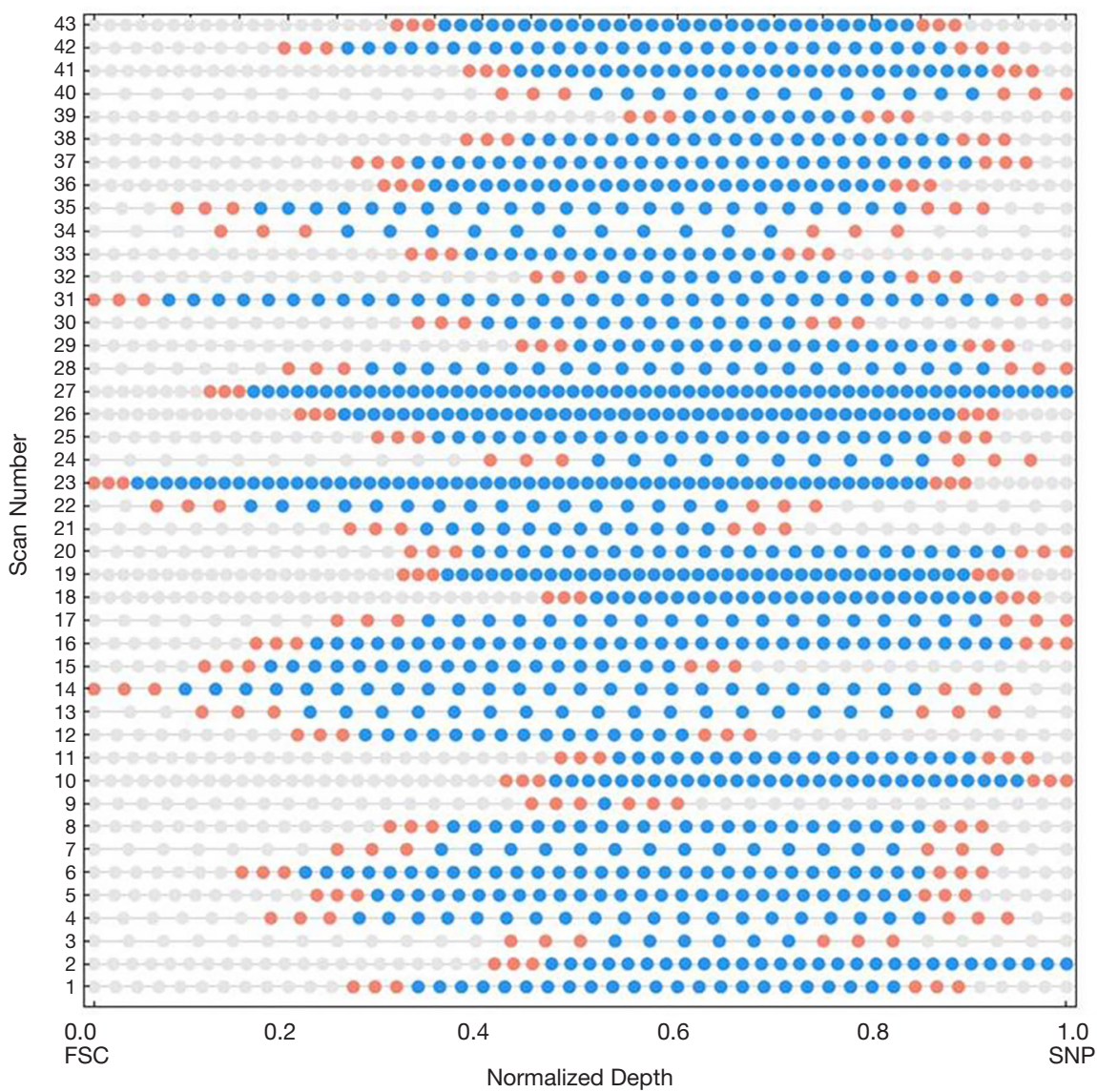

Figure 4 Overview of the data collected for the study and used for quantification: The epithelial thickness was normalized to 1 starting from the first superficial cell (FSC) [0] to the center of the SNP [1]. The definition of start [0] and end [1] point was done by the investigator. Differences in epithelial thickness are causing non-equidistant interslice distance. Blue points represent image slices which have been assigned to the layers of the epithelium with certainty by the algorithm software and which were available for automated quantification. Orange points represent the three image safety margin slices applied by the software and grey points represent image slices which could not be detected by the software with certainty and which were not available for quantification.

were categorized into SCs, intermediate cells and basal cells according to their functionality and morphology. During both, homeostasis and wound healing, the corneal epithelium is in a constant state of self-renewing and a complete turnover occurs in approx. 5 to 7 days. The epithelium is thereby maintained by basal cells which are the source of wing and SCs and a delicate balance of shedding followed by proliferation is critical in maintaining a smooth and uniform epithelial surface (24).

SCs are characterized by a polygonal cell pattern, bright illuminated cytoplasm, reflecting nuclei and perinuclear dark halo (25) and appear brighter in CLSM images than cells towards the posterior of the cornea (26). About $1 / 7$ of these cells are lost by desquamation within 24 hrs with a precedent change in their optical characteristics (25) with the darker ones being those about to desquamate from the surface (27). The intermediate layer is composed of wing cells. These cells form a regular mosaic of cells with sharp and reflecting cell borders showing the same reflectivity as SCs $(8,28)$. However, their cell nuclei are not well defined and the cytoplasm appears dark (24). They are smaller in size when compared to SCs but more regular in form, and they can be further subdivided according to their position into upper and lower wing cells, with the latter being smaller. The innermost part of the epithelium is formed by a monolayer of basal cells. These cells show the smallest diameter when compared to the layers above and have a cylindrical, columnar form $(25,28)$. They display a uniformly 

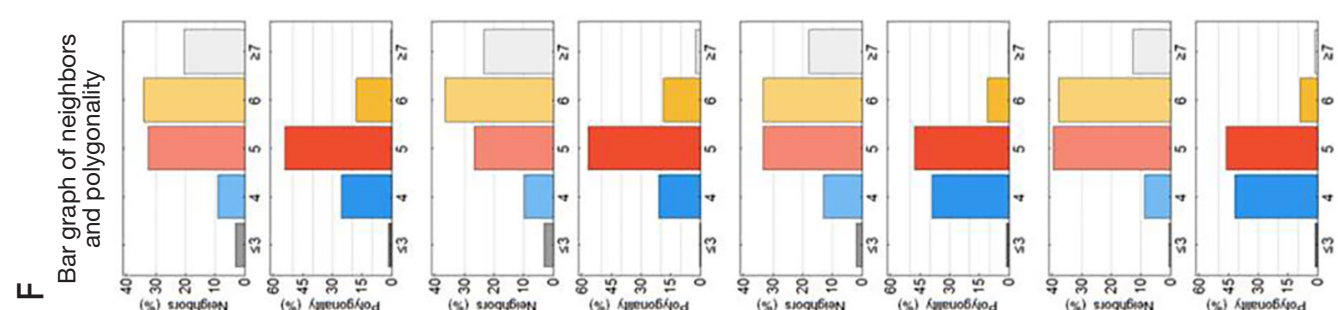

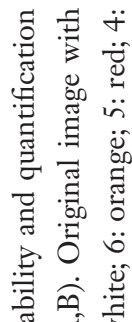
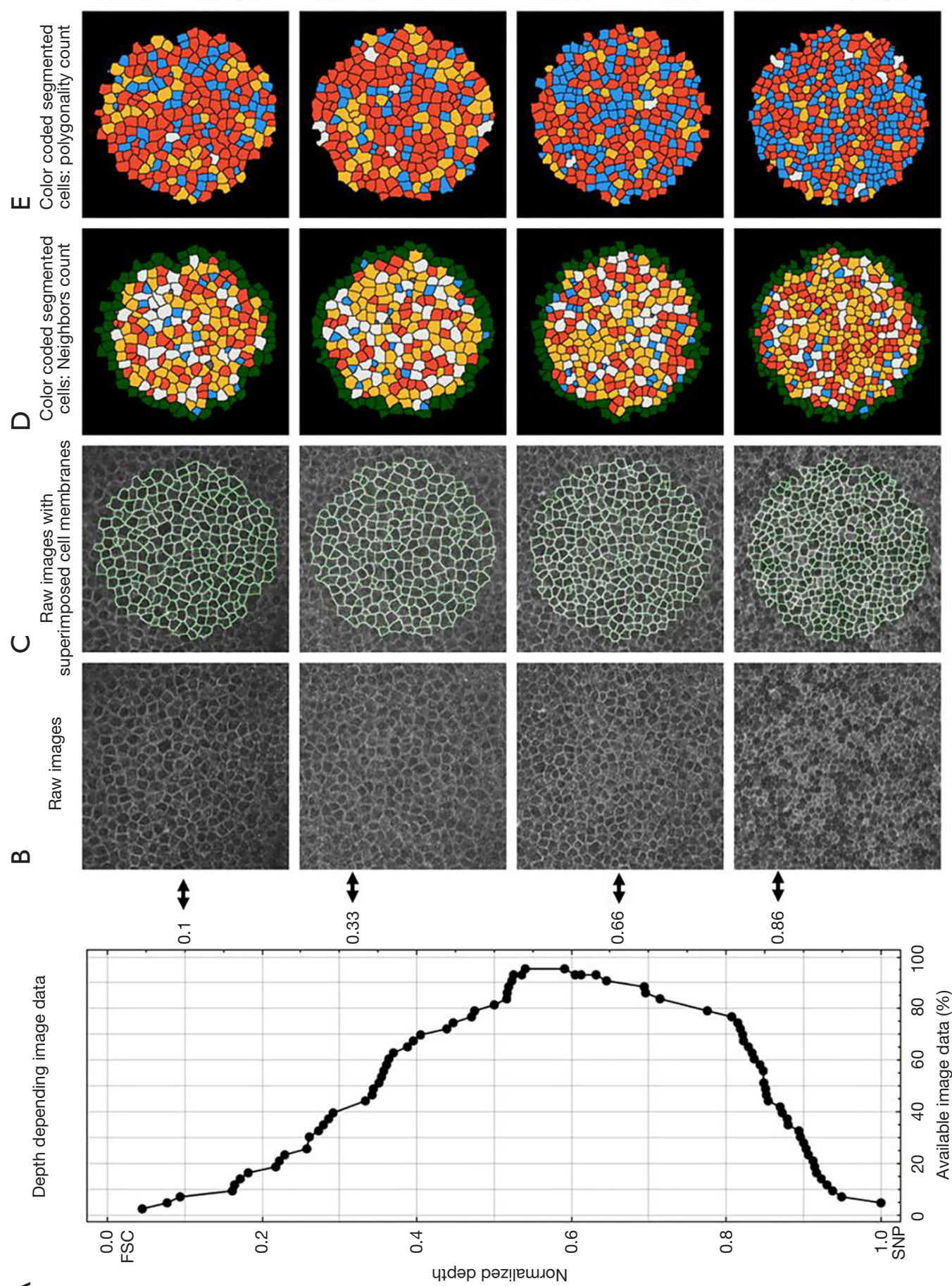

$\varangle$

ułdəp pəz!||euxoN

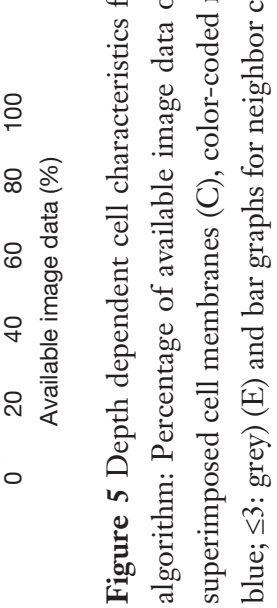


A
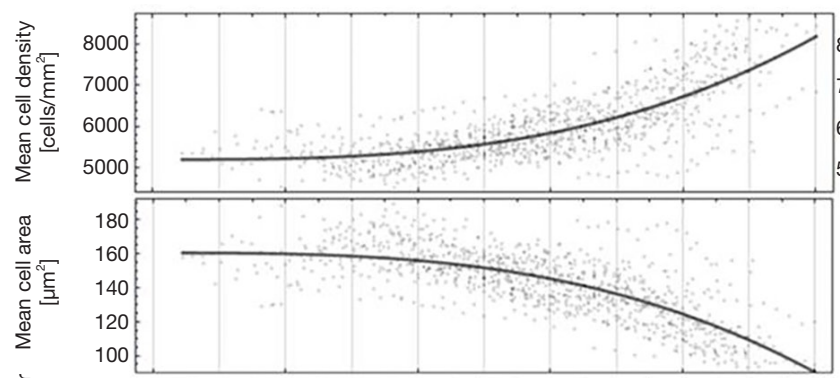

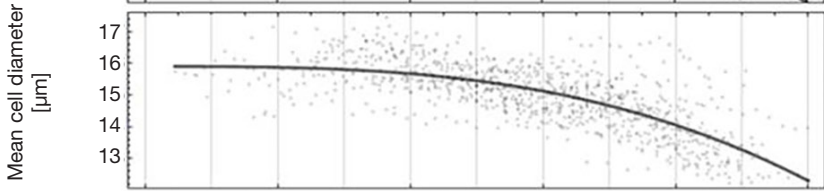
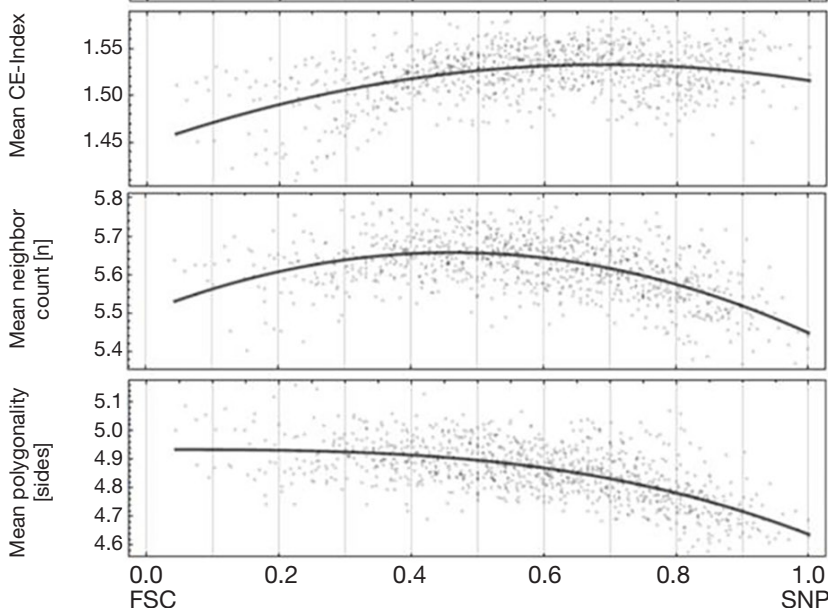

B
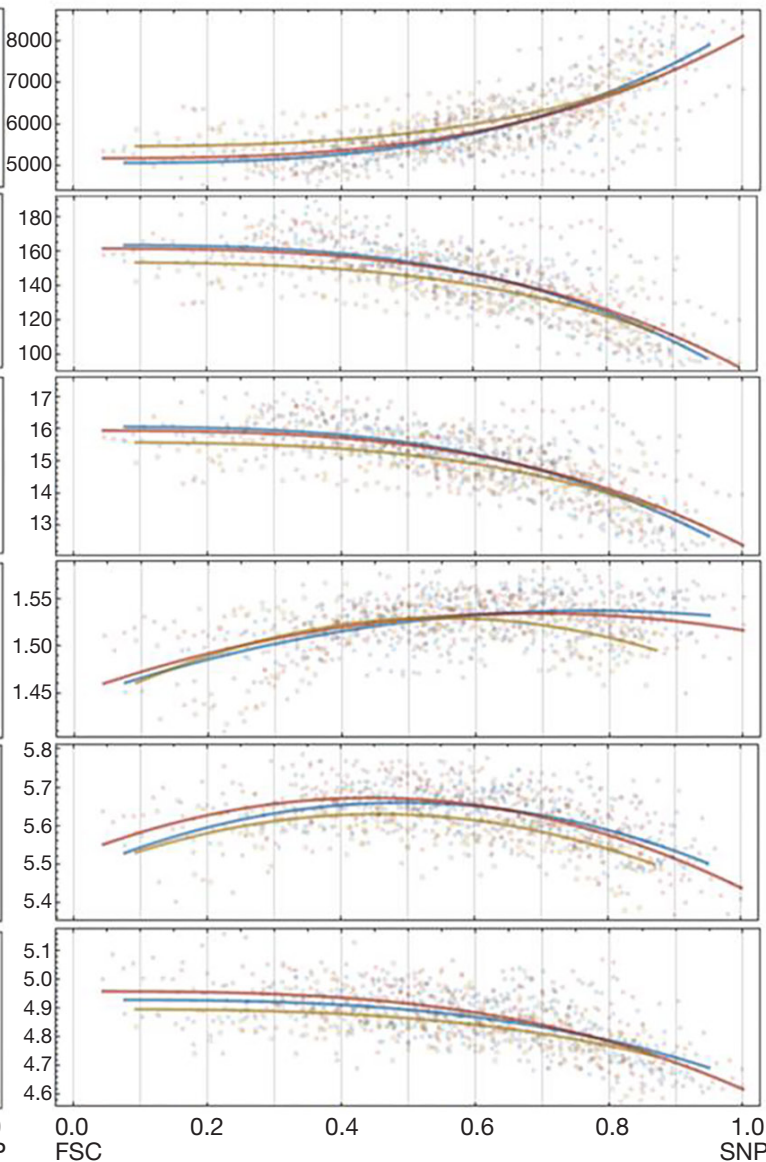

Figure 6 Characteristic quantities of corneal epithelial structures. Left column from top to bottom: mean of cell density, cell area, cell diameter, CE-Index, neighbor count and polygonality plotted against normalized scale. Right column from top to bottom: same parameters as left, but for different age groups. Age group definition was as follows: 20-25 years (orange); 36-55 years (olive); 56-70 years (blue).

Table 1 Summary of quantified epithelial cell parameters

\begin{tabular}{ll}
\hline Parameters & Definition \\
\hline Cell density & Number of cells per $\mathrm{mm}^{2}\left(\mathrm{cells} / \mathrm{mm}^{2}\right)$ \\
Cell area & Number pixels $\mathrm{x}$ area of a pixel $\left(\mu \mathrm{m}^{2}\right)$ \\
Cell diameter & Length of a straight line passing through the center of a cell $(\mu \mathrm{m})$ \\
CE-Index & Pattern of cell centroids \\
Neighbor count & Number of adjacent neighbors of each cell \\
Polygonality & Number of sides of a cell \\
\hline
\end{tabular}

Cross-sections of epithelial cells were quantified using appropriate image analysis algorithms. 
Table 2 Interpolated values of epithelial cell parameters

\begin{tabular}{|c|c|c|c|c|c|c|}
\hline $\begin{array}{l}\text { Interval position } \\
\text { normalized depth }\end{array}$ & $\begin{array}{l}\text { Mean cell density } \\
\quad\left(\text { cells } / \mathrm{mm}^{2}\right)\end{array}$ & $\begin{array}{l}\text { Mean cell area } \\
\left(\mu \mathrm{m}^{2}\right)\end{array}$ & $\begin{array}{c}\text { Mean cell } \\
\text { diameter }(\mu \mathrm{m})\end{array}$ & Mean CE-Index & $\begin{array}{l}\text { Mean neighbor } \\
\text { count }(n)\end{array}$ & $\begin{array}{c}\text { Mean polygonality } \\
\text { (sides) }\end{array}$ \\
\hline 0 & $5,197.02$ & 160.51 & 15.90 & 1.45 & 5.50 & 4.93 \\
\hline 0.2 & $5,220.97$ & 159.95 & 15.87 & 1.49 & 5.61 & 4.93 \\
\hline 0.5 & $5,571.19$ & 151.74 & 15.45 & 1.53 & 5.66 & 4.90 \\
\hline 0.6 & $5,843.59$ & 145.35 & 15.12 & 1.53 & 5.64 & 4.87 \\
\hline 0.7 & $6,223.75$ & 136.43 & 14.67 & 1.53 & 5.62 & 4.83 \\
\hline
\end{tabular}

Cross-sections of epithelial cells were quantified using appropriate image analysis algorithms. The scale between first superficial cell (FSC) to subbasal nerve plexus (SNP) was normalized from 0 to 1 ( $F S C=0, S N P=1$; increment: 0.1 ).
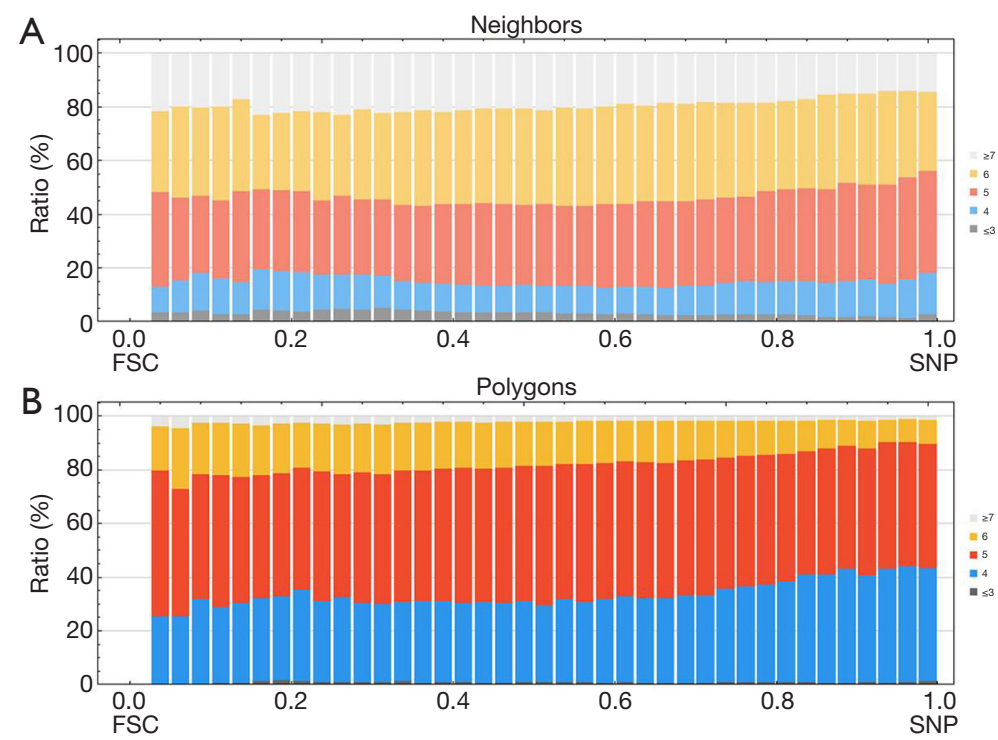

Figure 7 Distribution of neighbor count (A) and polygonality (B): stacked bar-charts show the variability in percentage of distribution from anterior to posterior for all analyzed scans.

bright cell border with dark cytoplasmic mass (27). Besides stem cells and transient amplifying cells, they are the only corneal epithelial cells capable of mitosis (29). During the renewing process of the corneal epithelium, the basal cells differentiate and migrate vertically towards the anterior surface to repopulate the cornea (30).
The present study represents a further development of a fully automated software tool for epithelial cell quantification. Compared to our former publication (13), the detection of epithelial cells was improved due to global brightness equalization and local structural contrast amplification in combination with bandpass- 
based membrane detection. The segmentation results reveal that efforts for further improvements on cell segmentation should be focused at the challenging regions of the superficial and deep sections of basal cells in order to provide automated cell characterization for the entire extent of the corneal epithelium. In addition to the cell features examined in the previous study (13), we have also introduced new parameters including density, aggregation (CE-Index), the count of surrounding cells and the polygonality of cells.

The mean cell densities gradually increased from anterior to posterior corresponding to the above-mentioned characteristics of the three different cell zones of the epithelium. Starting from the surface with the SCs and moving towards to the SNP, the cell morphology changes in size and shape from large but flat polygonal cells (SCs) to thin but long cylindrical cells (basal cells) and consequently their density in cross-sections increases. Comparison of our results with Eckard et al. (26) showed a general agreement regarding intermediate and basal cell layers. It must be mentioned that in our study the algorithm did not work sufficiently for SCs and the lowest parts of the basal cells located directly above the Bowman's membrane where the highest density is expected $(8,25)$. Regarding cell area, Prakasam et al. (13) reported a linear decrease from anterior to posterior which could be confirmed in this study while similarities and differences with other studies have already been discussed there. The minimal differences within the basal cell area were probably caused by the fact, that in the former evaluation the membranes were segmented comparatively thicker due to lower image quality and thus the minimum size was slightly lower.

The comparison of the herein performed manual count $v s$. the segmentation algorithm showed similar values in the depth range between 27 and $47 \mu \mathrm{m}$ of the analyzed scan representing the wing cell area and the upper part of the basal cells. Furthermore, the manually determined values were in accordance with other published works like by Gaujoux et al. (11).

The analyzed mean cell diameters showed a gradual decrease from anterior to posterior thereby showing comparable results to our previous study (13). When the herein performed manual count is compared with the segmentation algorithm, the values are comparable within the wing cells and could show comparable trends to former studies as well $(13,25,31)$.

The analyzed CE-Index showed a gradual increase from anterior to posterior displaying a regular distribution of the cells. In future studies, it would be important to evaluate whether this parameter also changes in pathologies of the corneal epithelium.

Regarding the number of neighbors surrounding a cell, the analysis demonstrated depth-dependent changes. In the majority, cells were surrounded by either 5 or 6 neighbors and fluctuations were detected in the front part and further downstream from point 0.75 towards 1 within the normalized depth. The variations in the anterior section may have been caused by the limited data available while the percentage of available image data within middle and posterior section accounted for between $40 \%$ to $90 \%$. It appears that there was a structural change in the section 0.75 of the normalized depth, i.e., the transition from lower wing cells to basal cells.

The polygonality of cells decreased from anterior to posterior confirming the previously described characteristic of the epithelial cell shapes changing from polygonal to cylindrical cells. The predominant shape of the cells was pentagonal with the relative amount decreasing towards the SNP. The second-most dominant shape was represented by quadrangular cells. Here the percentage remained stable at $30 \%$ while after section 0.75 of the normalized depth the relative amount rose to a maximum of $45 \%$ indicating the transition zone between lower wing cells and basal cells. However, it must be considered that SCs are the only cells that can be displayed in their entirety. In contrast, wing cells are arranged like roof tiles and with only part of their cell surface accessible in one section, thus influencing the analysis of all the above-mentioned parameters. Nevertheless, within this study we were able to present normative data for future comparison with data analyzed in pathologies of the corneal epithelium.

We have divided our subjects into three age groups whereas no age dependency was found. However, there are studies reporting age dependency in the human cornea. Zheng et al. examined 80 subjects and found agerelated changes in peripheral basal epithelial cell density and in peripheral basal endothelial cell density. It must be mentioned that a significance was only found within the youngest age group (32). Gambato et al. (33) reported an increase in cell diameter of superficial epithelial cells with subject age while basal epithelium and the SNP did not change depending on age in the 108 subjects studied (33). On the other hand, based on a study with 45 subjects, Mustonen et al. (34) reported no statistically significant correlation between superficial and basal epithelial cell density or age. Furthermore, no statistically significant differences 
in cell densities or cell areas of any corneal layer between gender nor between left or right eyes were reported (34). Eckard et al. (26) reported no statistically significant relationship between cell densities and age, sex or refraction as well in 68 subjects examined. In summary, all these studies mentioned above show controversial results.

It should be considered that our number of analyzed subjects was smaller when compared with all of the abovementioned studies. The question of whether a larger number of test persons examined further increases the significance of the normative data cannot be answered unequivocally. On the one hand, it can be assumed that an increased number of subjects improves the significance, but on the other, we postulate that newly determined quantities would be within the already determined quantity ranges. From our point of view, it is imperative to first improve the imaging strategy and the cell segmentation tools for superficial and deeper basal cell sections and then, in a second step, to increase the size of the normative cohort with these improved tools. However, the segmentation of SCs requires completely different tools that have yet to be developed.

As an outlook to possible future algorithmic developments, the $3 \mathrm{D}$ reconstructions could be potentially used for 3D characterization by volumetric cell features. However, even though the $3 \mathrm{D}$ reconstruction process achieves good structural coherence between adjacent images, the anisotropic optical resolution of the CLSM volumes presents a challenge for the segmentation and quantification algorithms. We have an almost isotropic voxel resolution of $0.91 \mu \mathrm{m} \times 0.91 \mu \mathrm{m} \times 1 \mu \mathrm{m}$ per voxel, but the optical resolution differs significantly between the z-direction (about $4 \mu \mathrm{m}$ ) and the en-face directions (about $1 \mu \mathrm{m})(35)$. Due to this, a reliable 3D segmentation and quantification of epithelial cells based on current data was not feasible.

This in vivo study demonstrates a CLSM-based imaging pipeline for cellular characterization of the human corneal epithelium. The concave contact cap and the integration of a second, large travel-range piezo actuator significantly improved the usability of this method. Furthermore, characteristic quantities of different epithelial cell layers were presented, whereas no age dependency was found. Given the growing interest and the importance of understanding the cellular changes in ophthalmological disorders or disorders which were described to affect cornea's morphology as well, a non-invasive in vivo CLSMbased imaging and quantification pipeline could serve as a powerful diagnostic tool and thereby improve the evaluation of therapeutic regimens. Changes in the herein analyzed parameters could be evaluated over longer periods of time and help in the understanding of underlying biological processes such as inflammation and wound healing. Moreover, longitudinal analyses of these parameters could help in the assessment of treatment efficacy and regeneration of the cornea. The data gained herein could serve as a first step to generate more normative epithelial data towards further investigations. Further significant effort is necessary to improve the algorithm for superficial and basal cell segmentation.

\section{Acknowledgments}

Funding: Parts of this work were supported by the Deutsche Forschungsgemeinschaft (DFG, German Research Foundation) - grant numbers STA 543/6-2 and KO 5003/12 - and by the German Federal Ministry of Education and Research (Antifibrotix, 03VP06230; RESPONSE "Partnership for Innovation in Implant Technology").

\section{Footnote}

Conflicts of Interest: All authors have completed the ICMJE uniform disclosure form (available at http:// dx.doi.org/10.21037/qims-20-1052). Dr. KAS reports grants from German Federal Ministry of Education and Research, during the conduct of the study; Dr. KS reports grants from Deutsche Forschungsgemeinschaft (DFG, German Research Foundation), during the conduct of the study; Dr. TS reports grants from German Federal Ministry of Education and Research, during the conduct of the study; Dr. SA reports grants from Deutsche Forschungsgemeinschaft (DFG, German Research Foundation), during the conduct of the study; Dr. BK reports grants from Deutsche Forschungsgemeinschaft (DFG, German Research Foundation), during the conduct of the study; SB reports grants from Deutsche Forschungsgemeinschaft (DFG, German Research Foundation), during the conduct of the study. The other authors have no conflicts of interest to declare.

Ethical Statement: The study protocol was approved by the Institutional Review Board [Rostock University Medical Center Ethics Committee, Registration number: A 20180162 (dated 23 August 2018)]. All study procedures followed the tenets of the Declaration of Helsinki, and informed 
consent was obtained from all subjects before enrollment.

Open Access Statement: This is an Open Access article distributed in accordance with the Creative Commons Attribution-NonCommercial-NoDerivs 4.0 International License (CC BY-NC-ND 4.0), which permits the noncommercial replication and distribution of the article with the strict proviso that no changes or edits are made and the original work is properly cited (including links to both the formal publication through the relevant DOI and the license). See: https://creativecommons.org/licenses/by-nc-nd/4.0/.

\section{References}

1. Bille JF, editor. High Resolution Imaging in Microscopy and Ophthalmology: New Frontiers in Biomedical Optics. Springer International Publishing; 2019.

2. Bizheva K, Tan B, MacLelan B, Kralj O, Hajialamdari M, Hileeto D, Sorbara L. Sub-micrometer axial resolution OCT for in-vivo imaging of the cellular structure of healthy and keratoconic human corneas. Biomed Opt Express 2017;8:800-12.

3. Werkmeister RM, Sapeta S, Schmidl D, Garhöfer G, Schmidinger G, Aranha Dos Santos V, Aschinger GC, Baumgartner I, Pircher N, Schwarzhans F, Pantalon A, Dua H, Schmetterer L. Ultrahigh-resolution OCT imaging of the human cornea. Biomed Opt Express 2017;8:1221-39.

4. Tan B, Hosseinaee Z, Han L, Kralj O, Sorbara L, Bizheva K. $250 \mathrm{kHz}, 1.5 \mu \mathrm{m}$ resolution SD-OCT for invivo cellular imaging of the human cornea. Biomed Opt Express 2018;9:6569-83.

5. Petroll WM, Robertson DM. In Vivo Confocal Microscopy of the Cornea: New Developments in Image Acquisition, Reconstruction, and Analysis Using the HRTRostock Corneal Module. Ocul Surf 2015;13:187-203.

6. Erie JC, McLaren JW, Patel SV. Confocal Microscopy in Ophthalmology. Am J Ophthalmol 2009;148:639-46.

7. Zhivov A, Guthoff RF, Stachs O. In vivo confocal microscopy of the ocular sur-face: from bench to bedside and back again. Br J Ophthalmol 2010;94:1557-8.

8. Zhivov A, Stachs O, Kraak R, Stave J, Guthoff RF. In vivo confocal microscopy of the ocular surface. Ocul Surf 2006;4:81-93.

9. Al-Aqaba MA, Dhillon VK, Mohammed I, Said DG, Dua HS. Corneal nerves in health and disease. Prog Retin Eye Res 2019;73:100762.

10. Kokot J, Wylęgała A, Wowra B, Wójcik Ł, Dobrowolski
D, Wylęgała E. Corneal confocal sub-basal nerve plexus evaluation: a review. Acta Ophthalmol 2018;96:232-42.

11. Gaujoux T, Touzeau O, Laroche L, Borderie VM. Morphometry of corneal ep-ithelial cells on normal eyes and after anterior lamellar keratoplasty. Cornea 2010;29:1118-24.

12. Bohn S, Sperlich K, Allgeier S, Bartschat A, Prakasam R, Reichert KM, Stolz H, Guthoff R, Mikut R, Köhler B, Stachs O. Cellular in vivo 3D imaging of the cornea by confocal laser scanning microscopy. Biomed Opt Express 2018;9:2511-25.

13. Prakasam RK, Winter K, Schwiede M, Allgeier S, Zhivov A, Köhler B, Guthoff RF, Stachs O. Characteristic quantities of corneal epithelial structures in confocal laser scanning microscopic volume data sets. Cornea 2013;32:636-43.

14. Le Q, Xu J, Deng SX. The diagnosis of limbal stem cell deficiency. Ocul Surf 2018;16:58-69.

15. Chan EH, Chen L, Rao JY, Yu F, Deng SX. Limbal Basal Cell Density Decreases in Limbal Stem Cell Deficiency. Am J Ophthalmol 2015;160:678-84.e4.

16. Chang PY, Carrel H, Huang JS, Wang IJ, Hou YC, Chen WL, Wang JY, Hu FR. Decreased density of corneal basal epithelium and subbasal corneal nerve bundle changes in patients with diabetic retinopathy. Am J Ophthalmol 2006;142:488-90.

17. Bowers NR, Boehm AE, Roorda A. The effects of fixational tremor on the ret-inal image. J Vis 2019;19:8.

18. Martinez-Conde S, Macknik SL, Hubel DH. The role of fixational eye move-ments in visual perception. Nat Rev Neurosci 2004;5:229-40.

19. Allgeier S, Zhivov A, Eberle F, Koehler B, Maier S, Bretthauer G, Guthoff RF, Stachs O. Image reconstruction of the subbasal nerve plexus with in vivo confocal microscopy. Invest Ophthalmol Vis Sci 2011;52:5022-8.

20. Ramer U. An iterative procedure for the polygonal approximation of plane curves. Comput Graph Image Process 1972;1:244-56.

21. Douglas DH, Peucker TK. Algorithms for the reduction of the number of points required to represent a digitized line or its caricature. Cartogr Int J Geogr Inf Geo-visualization 1973;10:112-22.

22. Clark PJ, Evans FC. Distance to Nearest Neighbor as a Measure of Spatial Re-lationships in Populations. Ecology 1954;35:445-53.

23. Guthoff RF, Baudouin C, Stave J. General Anatomical Considerations. In: Atlas of Confocal Laser Scanning Invivo Microscopy in Ophthalmology. Berlin, Heidel-berg: 
Springer, 2006.

24. Levin LA, Nilsson SFE, Ver Hoeve J, Wu SM, Kaufman PL. Cornea and Sclera. In: Adler's Physiology of the Eye E-Book. Elsevier Health Sciences, 2011.

25. Guthoff RF, Zhivov A, Stachs O. In vivo confocal microscopy, an inner vision of the cornea - a major review. Clin Exp Ophthalmol 2009;37:100-17.

26. Eckard A, Stave J, Guthoff RF. In vivo investigations of the corneal epithelium with the confocal Rostock Laser Scanning Microscope (RLSM). Cornea 2006;25:127-31.

27. Tavakoli M, Hossain P, Malik RA. Clinical applications of corneal confocal microscopy. Clin Ophthalmol 2008;2:435-45.

28. Guthoff RF, Stave J. In Vivo Micromorphology of the Cornea: Confocal Mi-croscopy Principles and Clinical Applications. In: Reinhard T., Larkin D. (eds) Cornea and External Eye Disease. Essentials in Ophthalmology. Berlin, Heidelberg: Springer, 2006;173-208.

29. DelMonte DW, Kim T. Anatomy and physiology of the cornea. J Cataract Refract Surg 2011;37:588-98.

Cite this article as: Sterenczak KA, Winter K, Sperlich K, Stahnke T, Linke S, Farrokhi S, Klemm M, Allgeier S, Köhler B, Reichert KM, Guthoff RF, Bohn S, Stachs O. Morphological characterization of the human corneal epithelium by in vivo confocal laser scanning microscopy. Quant Imaging Med Surg 2021;11(5):1737-1750. doi: 10.21037/qims-20-1052
30. Eghrari AO, Riazuddin SA, Gottsch JD. Overview of the Cornea: Structure, Function, and Development. Prog Mol Biol Transl Sci 2015;134:7-23.

31. Beuerman RW, Pedroza L. Ultrastructure of the human cornea. Microsc Res Tech 1996;33:320-35.

32. Zheng T, Le Q, Hong J, Xu J. Comparison of human corneal cell density by age and corneal location: an in vivo confocal microscopy study. BMC Ophthalmol 2016;16:109.

33. Gambato C, Longhin E, Catania AG, Lazzarini D, Parrozzani R, Midena E. Aging and corneal layers: an in vivo corneal confocal microscopy study. Graefes Arch Clin Exp Ophthalmol 2015;253:267-75.

34. Mustonen RK, McDonald MB, Srivannaboon S, Tan AL, Doubrava MW, Kim CK. Normal human corneal cell populations evaluated by in vivo scanning slit confocal microscopy. Cornea 1998;17:485-92.

35. Heidelberg Retina Tomograph 2 Operating Instructions of Software Ver-sion 1.1. Dossenheim Germany: Heidelberg Engineering GmbH, 2004. 